\title{
Performance Evaluation of Different Mobile Ad-hoc Network Routing Protocols in Difficult Situations
}

\author{
Sultan Mohammed Alkahtani ${ }^{1}$, Fahd Alturki ${ }^{2}$ \\ Electrical Engineering Department, King Saud University, Riyadh, Saudi Arabia
}

\begin{abstract}
Performance evaluation of Mobile Ad-hoc Network (MANET) routing protocols is essential for selecting the appropriate protocol for the network. Many routing protocols and different simulation tools were proposed to address this task. This paper will introduce an overview of MANETs routing protocols as well as evaluate MANET performance by using three reactive protocols-Dynamic Source Routing (DSR), AdHoc On-demand Distance Vector (AODV), and Dynamic MANET On-Demand (DYMO) - in three different scenarios. These scenarios are designed carefully to mimic real situations by using OMNET++. The first scenario evaluates the performance when the number of nodes increases. In the second scenario, the performance of the network will be evaluated in the presence of obstacles. In the third scenario, a group of nodes will be suddenly shut down during the communication. The network evaluation is carried out in terms of packets received, end-to-end delay, transmission count or routing overhead, throughput, and packet ratio.
\end{abstract}

Keywords-Ad-hoc network; performance evaluation; network simulation; MANET; DSR; AODV; DYMO; routing protocols; Omnet++

\section{INTRODUCTION}

Wireless networks have reduced the use of wired networks by enabling devices to communicate easily without using cables and wires. The free mobility that is provided by wireless networks has overcome some of the challenges of wired networks such as being difficult, time-consuming, and expensive to install and maintain. These networks have become more popular and secure with new technologies and security protocols [1].

A wireless network is divided into two main types: infrastructure-based and infrastructure-less. An infrastructurebased network is one in which all wireless devices communicate and share information through infrastructure units such as access points, routers, or PCs running access point software [2]. Infrastructure-less or ad-hoc networks are direct connections between wireless devices with no infrastructure units such as a router or access point. Fig.1 compares how devices are connected in an infrastructure network versus an infrastructure-less network. In ad-hoc networks, the network can be set up easily with no aid from infrastructure units, such as access points. In this network, each node has a transmission range. When the destination is out of this range, the connectivity between the transmitter and the receiver will depend on the intermediate nodes. In the aforementioned case, the intermediate node will act as a router. This independence in an ad-hoc network offers free deployment and low cost for the network. Ad-hoc networks can be classified into three types: Mobile Ad-hoc Networks (MANETs), Wireless Sensor Networks (WSNs), and Wireless Mesh Networks (WMNs).

A MANET is a temporary auto-configuration network that supports users continuously and dynamically changes its network topology. This means the nodes communicate without administration and may connect to the Internet or operate as standalones. In MANETs, routing protocols are essential to finding the right path between the source and the destination. There are many challenging factors for the routing protocols of MANETs. In some situations, the mobility of the nodes may create a significant challenge to the routing protocol because some of the nodes will be out of the transmission range, which will require finding an alternative path to the destination. In a real network, the node is affected by power constraints, as the node is operating with a limited battery, which will affect the lifetime of the nodes [3].

This paper will evaluate the performance of three reactive protocols in three different scenarios. These scenarios are designed to evaluate the network performance under varying situations. The first scenario resembles a standard case, without shutdowns or obstacles. The scenario captures the MANET performs as the number of nodes in the network increases. The second scenario tests the three protocols in the presence of obstacles. The third scenario evaluates protocol performance under random shutdown conditions. In the following sections, the protocols are discussed in more detail and existing literature is reviewed. Following this is a more detailed discussion of the simulation used, the different scenarios, and finally the results and conclusion.

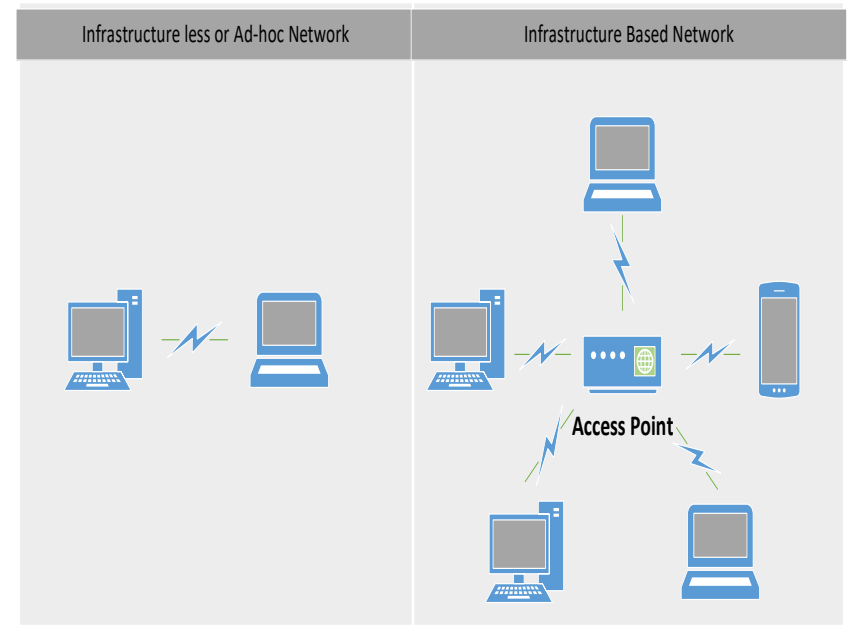

Fig. 1. Ad-hoc Network and Infrastructure Network. 


\section{MANET Routing PROTOCOLS}

MANET routing protocols may classify into three categories, as shown in Fig. 2: Reactive (On-demand), Proactive (Table-driven), and Hybrid [4],[5]. This work will focus on evaluating the performance of three types of reactive protocols: Dynamic Source Routing (DSR), Ad-hoc OnDemand Distance Vector (AODV), and Dynamic MANET On-Demand (DYMO).

\section{A. Dynamic Source Routing}

The DSR is one of the reactive protocols based on source routing algorithms. The selection of a path is the source's responsibility. It does so by initiating a request packet and sending it to its neighbors. The packet header will contain the information of all intermediate nodes or hops in the route until the destination is reached. The route that was recently discovered will be stored in the cache route of the node. The control message that is periodically exchanged in proactive is no longer used because it relays on the MAC layer to discover the failure of the link. Consequently, it has two advantages over proactive protocols, i.e., in terms of battery consumption and network overhead [6].

Two processes are used in the DSR protocol. The first is to discover the route from the source to the destination. The second is to maintain the route. Route discovery takes place when the source needs to communicate with a specific node. First, the source starts searching in its cache route for the route to the destination. If the source finds the route, it will communicate immediately. If it does not find the route in its cache route, the source will start to discover the route by flooding or broadcasting the route request packet to all neighboring nodes within the transmission range, and the source will add its information in the header of the request packet; the neighbors will search in their caches for the destination node. If one of them finds the path, the replay packet will be created and send to the source. If no such route is found in their cache route, each node will add its address to the request packet and rebroadcast to neighboring nodes within their transmission range until it reaches to the destination or the intermediate node that has information about the route to the destination. If the destination is not found within time to live (TTL), the packet will be expired, and the source will generate a new route request with an increased TTL value. Fig. 3 shows the process of route discovery, starting with initiation of the Routing Request (RREQ) and broadcasting it to the neighbors, finishing when it reaches the destination [7].

When the Routing Request (RREQ) packet reaches its destination, the destination node will create the Route Reply (RREP) and search for the route information to the source. If the destination finds the route, it will use it in the RREP. If there is no information about the path to the source in its cache route, it will use the same accumulation path in the RREQ. When the RREP reaches the source, it will save the route of the destination in the cache route and start communicating. The RREP could return in various ways and save each route information in its cache route [7].

Fig. 4 illustrates the RREP process. In the second process, which maintains the route, each node in the route is responsible for receiving and sending the data to the next hop. An acknowledgment can confirm the link capability to carry the data. The acknowledgment already exists in MAC protocols such as IEEE 802.11, which is a link layer acknowledgment frame. If no acknowledgment has been received, the sender node will consider the link to be "broken" and will remove this link from its route cache. It will then create the Route Error (RRER) and send it to each node that has sent a packet. Furthermore, the source will start to create RREQ and flood it again to select another path.

Fig. 5 illustrates the broken link between 3 and 6. In this case, node 3 receives the packet and transmits it to node 6 ; the acknowledgment is still not received in node 3 . Thus, node 3 will send an acknowledgment request to node 6. If the acknowledgment is still not received, the node will consider the link broken and create the RRER to send it to the source. The DSR protocol has an advantage over the proactive protocols because it requests the route only when it is needed. This feature will reduce the overhead of the network caused by the control messages and the bandwidth consumption will reduce. However, increasing the number of hops will increase the header of the Routing Request (RREQ) packet. It is possible to have so many routes to the destination in the cache route of the source when the number of nodes in the network is extensive. This may increase collisions of packets, which could cause congestion at the nodes in the case of sending a reply. This problem is called reply storms [8].

\section{MANETs Protocols}

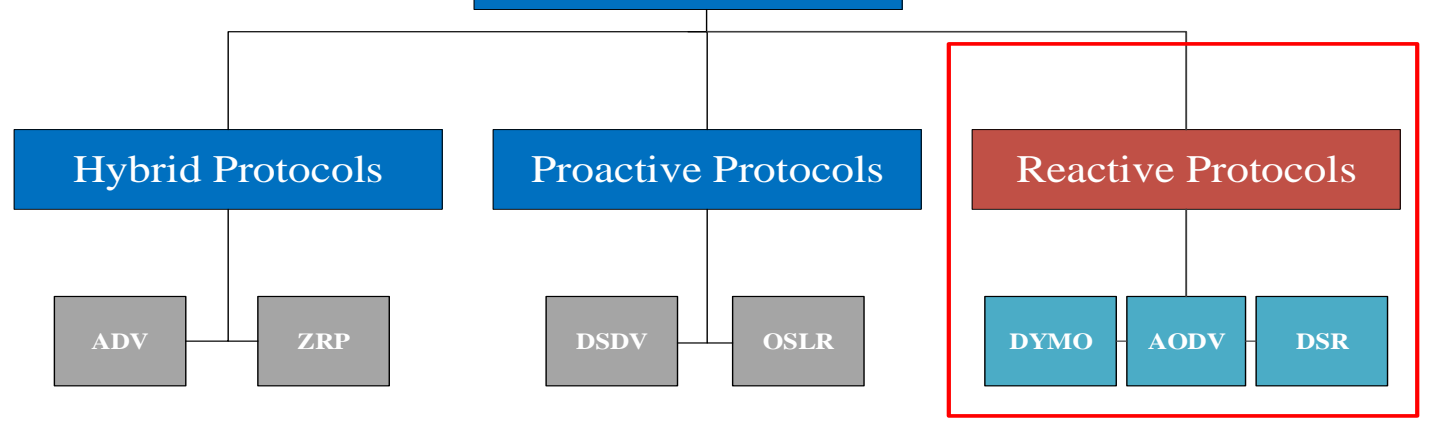

Fig. 2. Protocols Categories of MANETs. 


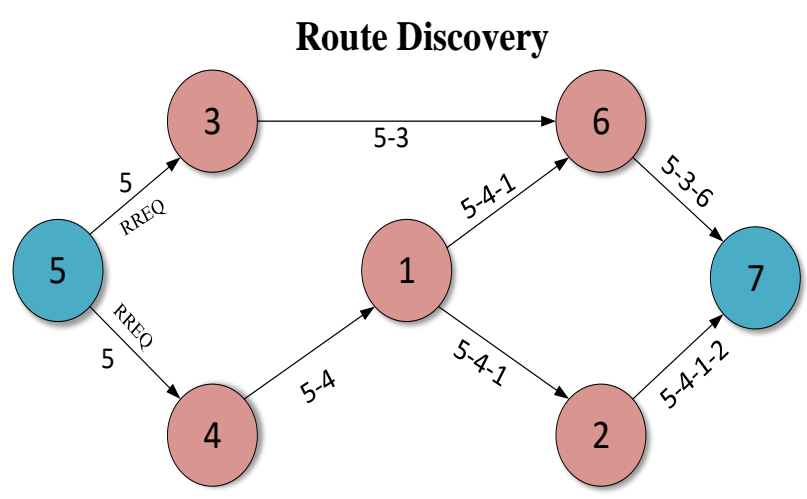

Fig. 3. Route Discovery Process (RREQ).

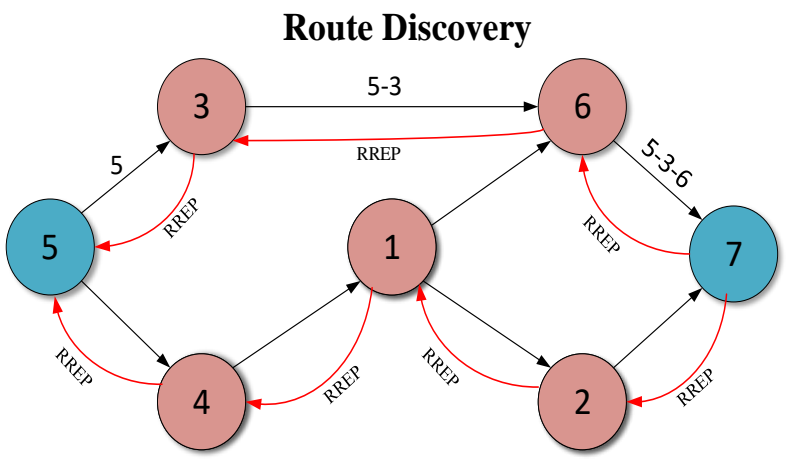

Fig. 4. Route Discovery Process (RREP).

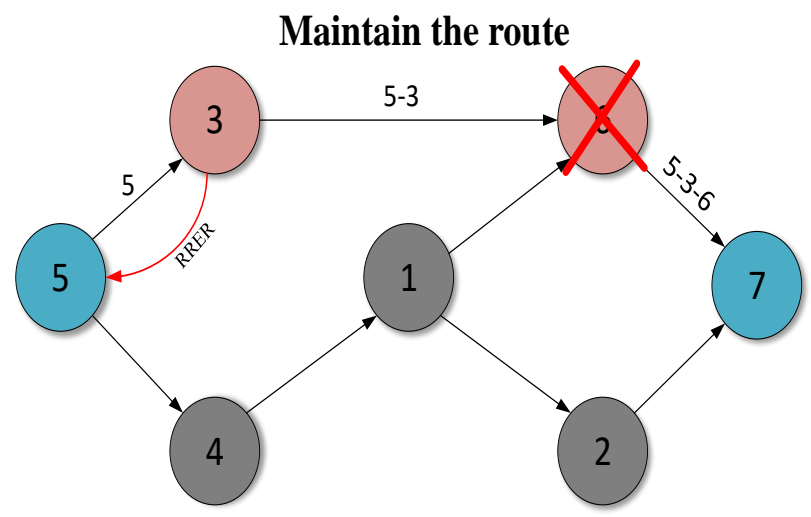

Fig. 5. Maintain the Route Process (RRER).

\section{B. Ad-hoc On-Demand Distance Vector}

The AODV is designed to follow the principle of the distance vector algorithm, which means each node has a routing table that is used to store the active paths. The ADOV mechanism is based on the same two processes as DSR, which are route discovery and maintaining the route. Moreover, AODV does not require the node to maintain the idle route. In AODV guarantee loop-free because each request packet has a unique ID number for each packet. This will result in eliminating the distance vector problem counting to infinity. In the route discovery process, the routes are determined when needed. When the source needs to communicate, it examines its own routing table for the route information of the destination. Each routing table consists of information, as shown in Table I.
TABLE I. ROUTING TABLE

\begin{tabular}{|l|}
\hline Routing Table \\
\hline Destination Address \\
\hline Next Hop Address \\
\hline Destination Sequence Number \\
\hline Hop Count \\
\hline
\end{tabular}

The destination sequence number is used to indicate the recent path and is created by the destination once RREQ is reached in the destination [9]. If a route is found in the routing table, then the source will start to send the packets to distention. Otherwise, the source will hold the message in the message queue and initiate RREQ; the RREQ will be flooded within the network until the RREQ reaches the destination. The RREQ contains information as shown in Table II [9].

The destination sequence number used to identify the new path and RREQ ID to avoid the loops. The source sequence number is a unique number used in the new RREQ. When RREQ arrives at an intermediate node, the routing table will be checked for route information. If there is none, the RREQ will continue the process, but the number of hops will be increased one to the previous hops until reach the destination. If the destination is not found within the TTL, the packet will expire and be deleted. The new packet will increase the TTL value. The source sequence number will be higher, and the nodes will update their routing table once they receive a higher source sequence number. In this case, the route is not part of the packet header. When the destination receives the RREQ, the RREP will be generated and sent back to the source. Each intermediate node will store the forward path to the routing table. The destination will be added to the list of active neighbors. Fig. 6 illustrates the route discovery process for AODV [10]. In maintaining the routing process, this process helps to preserve the routes when the topology changes by sending a Hello message between the nodes in the active route to verify the route's validity. When the response is missing, the node reports on the affected node by sending RRER contains unreachable node in the desired route. Then the route replay error will be sent to the source. When the source node receives the RRER, it will compare it with its routing table; the broken route will be deleted from the routing table and the source will generate a new RREQ to discover a new path [11].

TABLE II. ROUTE REQUEST PACKET (RREQ)

\begin{tabular}{|l|}
\hline Route request (RREQ) \\
\hline RREQ ID \\
\hline Source IP Address \\
\hline Source Sequence Number \\
\hline Destination IP Address \\
\hline Destination Sequence Number \\
\hline Hop Count \\
\hline Time to Live (TTL) \\
\hline
\end{tabular}




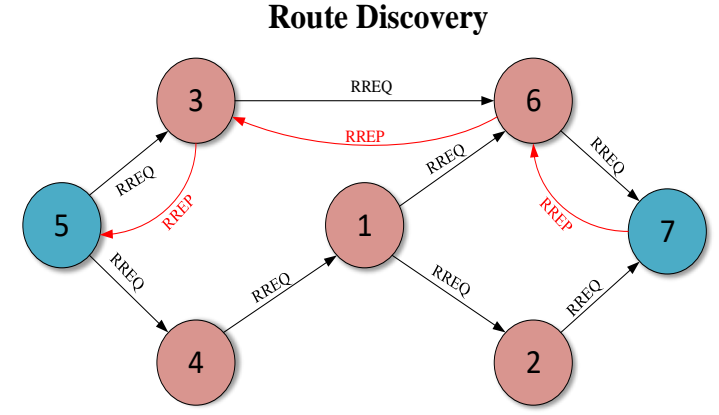

Fig. 6. Route Discovery Process (RREQ and RREP) for AODV.

\section{Dynamic MANET On-Demand}

The Dynamic MANET On-Demand (DYMO) is a combination of AODV and DSR features. The DYMO has two main processes: route discovery and route maintenance. The route discovery process is used when there is no information about the route between the source and the destination in the routing table of the node. After the path is found, the maintain route process will take place. In route discovery, the source will search for the route information of the destination in the routing table. The routing table contains the destination address, the sequence number of the destination, the next hop address, and the hop count, with the same information as the routing table in AODV. When there is no information about the destination's route, in the routing table of the source, the Routing Request (RREQ) will be generated and broadcast across the network. When the intermediate node receives the RREQ, the node will search in the routing table; if the node did not find the target, will add itself to the RREQ and broadcast it. The idea of adding each node to its information in RREQ is to update the routing table of the next node. This guarantees that each routing table is updated in intermediate nodes. The route reply RREP will be created and sent back to the source once the destination or intermediate node finds the route to the target or the destination, as shown in Fig. 7. In maintaining the route process, DYMO uses the Hello message or beacon message to check the link validity as in AODV if the broken link is detected, the node will create a RERR message, add all the nodes in the broken link, and broadcast back to the intermediate nodes. The nodes that received the RRER will compare the record list in RRER with their routing table. If the sequence number of the destination is equal to or higher than the sequence number in the routing table, the information of the route will be deleted [12].

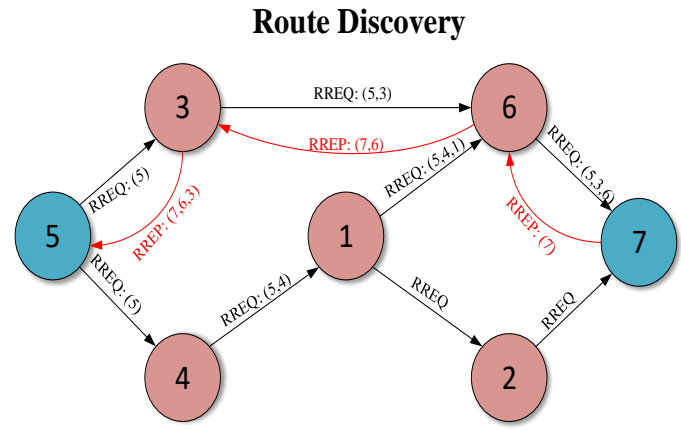

Fig. 7. Route Discovery Process (RREQ and RREP) for DYMO.

\section{RELATED WORKS}

Many published works have evaluated and tested the performance of MANET protocols in different scenarios and metrics. These works have used different simulation tools. Table III shows the recent research. In [13], two reactive protocols, DSR and AODV, were evaluated using the NS-2.35 simulator. The performance was evaluated under two metrics: packet delivery ratio and remaining energy in two scenarios. The scenarios used were changing the number of nodes in the network from 10,20,30,40, and 50 with increasing node velocity. The performance of DSR is better than that of AODV in both scenarios.

TABLE III. RELATED WORKS

\begin{tabular}{|c|c|c|c|}
\hline Year & Author & $\begin{array}{l}\text { Simulation } \\
\text { Tool }\end{array}$ & Scope of Work \\
\hline 2020 & $\begin{array}{l}\text { Jamilah, Amnah, } \\
\text { and K. Ibrahim }\end{array}$ & NS-2.35 & $\begin{array}{l}\text { Comparison between DSR } \\
\text { and AODV when the number } \\
\text { of nodes changed from } 10 \text {, } \\
20,30,40 \text {, and } 50 \text { with } \\
\text { increasing node velocity }\end{array}$ \\
\hline 2020 & $\begin{array}{l}\text { Russell, Nan } \\
\text { Wang, and } \\
\text { Daniel }\end{array}$ & NS-3 & $\begin{array}{l}\text { Comparison between AODV, } \\
\text { DSR, OLSR, and DSDV } \\
\text { when the velocity of the } \\
\text { nodes and the area size } \\
\text { increased }\end{array}$ \\
\hline 2020 & $\begin{array}{l}\text { Marwan, } \\
\text { Salama, } \\
\text { Hairulnizam, } \\
\text { Aida, Azizul, } \\
\text { Mustafa, and } \\
\text { Mohammed }\end{array}$ & NS-2 & $\begin{array}{l}\text { Comparison between three } \\
\text { protocols-AODV, DSDV, } \\
\text { and AOMDV-in terms of } \\
\text { metrics packet delivery ratio } \\
\text { and throughput }\end{array}$ \\
\hline 2020 & $\begin{array}{l}\text { A. S. Mustafa, } \\
\text { M. M. Al-Heeti, } \\
\text { M. M. Hamdi, } \\
\text { and A. M. } \\
\text { Shantaf }\end{array}$ & NS-2 & $\begin{array}{l}\text { Comparison between GPSR } \\
\text { and AODV when the size of } \\
\text { network varied in terms of } \\
\text { packet ratio, end-to-end } \\
\text { delay, and throughput. }\end{array}$ \\
\hline
\end{tabular}

In [14], the work showed a comparison of four different routing protocols-AODV, DSR, OLSR, and DSDV - in two scenarios. The two scenarios were varying the velocity of the nodes and the area size and comparing their performance in packet delivery ratio and end-to-end delay. The AODV has higher performance in packet delivery ratio in the case of a high velocity of nodes, while DSDV has the lowest packet delivery ratio. In terms of end-to-end delay, DSR has the largest end-end end delay, while the OLSR has the lowest end-to-end delay in both scenarios.

In [15], the authors presented a comparison between three protocols-AODV, DSDV, and AOMDV-in different metrics packet delivery ratios, throughputs, end to end delays, and packet loss ratios. The simulation time was in the range of 600 to 3400 seconds. The AOMDV performs better than the other protocols in terms of metrics packet delivery ratio, throughput, and packet loss ratios. However, the DSDV has the lowest end-to-end delay among the other protocols. Meanwhile, [16] presented a performance evaluation between Greedy Stateless Routing Perimeter (GPSR) and AODV when the size of network varied $500 \times 500,750 \times 750,1000 \times 1000$, $1250 \times 1250$, and $1500 \times 1500$ in terms of packet ratio, end-toend delay, and throughput. The number of nodes was 50, with 
a mobility of $20 \mathrm{~m} / \mathrm{s}$. GPSR performed better than AODV in terms of all three metrics. AODV suffered from high delay when the size of the network is 1500x1500. However, increasing the network size will degrade the performance of the protocol.

\section{Simulation}

This work evaluated and compared the performance of three protocols by using OMNET++. OMNET++ is an opensource that provides a free simulation environment for educational use with a wide variety of platforms. It has received a great amount of attention from researchers and developers. OMNET++ has different frameworks, modules, and components based on $\mathrm{C}++$; these are used primarily to build the networks. INETMANET provides modules in various layers that help to build the network and have additional modules and protocols, especially for MANETs, as compared to other frameworks [17].

\section{A. Node Implementation}

The node was implemented with a wireless network interface controller that supports 54 Mbps, as shown in Fig. 8. The MANET protocols are in the network layer, and the "manetrouting" module was used as a routing protocols pool with DSR, AODV, and DYMO, which will help to configure the network. In the transport layer, the UDP was used as a transport protocol. The UDPApp is the application providing a data packet that is 512B in size. The node has a transmission range that is coupled with a transmission power of $2 \mathrm{~mW}$; the receiving sensitivity is $-85 \mathrm{dBm}$.

\section{B. Network Implementation}

The network dimensions are $1000 \mathrm{~m} \times 1000 \mathrm{~m}$. The carrier frequency is $2.4 \mathrm{GHz}$ and each node has a transmission range that is coupled with the transmission power and receiving sensitivity of the node. The nodes have mass mobility with a velocity varying from 0.01 to 15 meters per second. Table IV summarizes the simulation setup of the network.

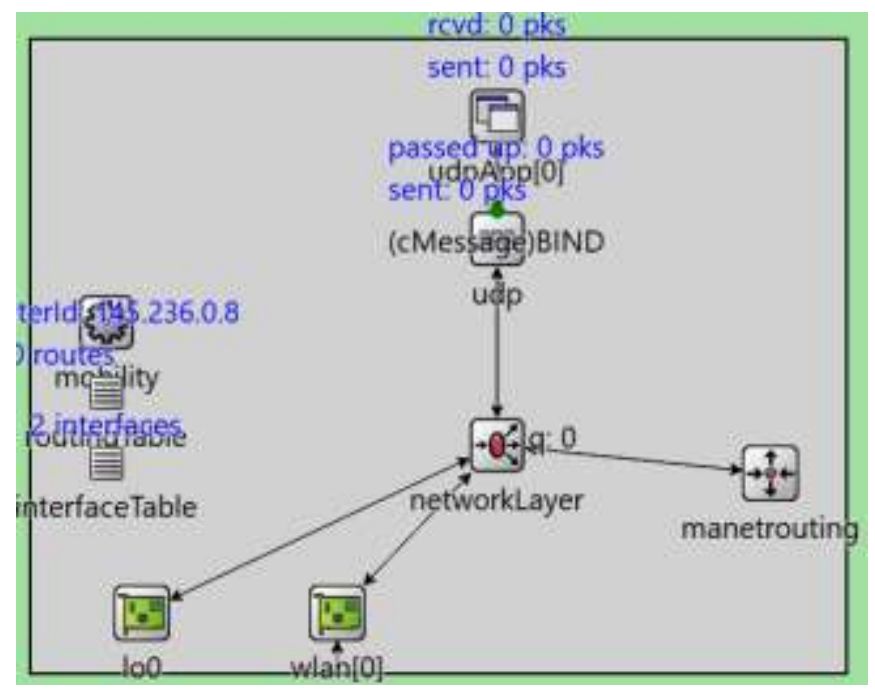

Fig. 8. Node Configuration.
TABLE IV. SIMULATION SETUP

\begin{tabular}{|l|l|}
\hline Parameters & Values \\
\hline Network Dimensions & $1000 \mathrm{~m} \mathrm{X} \mathrm{1000m}$ \\
\hline Carrier Frequency & $2.4 \mathrm{GHz}$ \\
\hline Radio Bitrate & $54 \mathrm{Mbps}$ \\
\hline Radio Transmission Power & $2 \mathrm{~mW}$ \\
\hline Receiving Sensitivity & $-85 \mathrm{dBm}$ \\
\hline Routing Protocols & DSR, AODV, and DYMO \\
\hline Number of Nodes & 25,45 \\
\hline Message Size & 512 Byte \\
\hline Node Mobility & Mass mobility $(0.01$ to 15$) \mathrm{m} / \mathrm{s}$ \\
\hline Simulation Time & $500 \mathrm{~s}$ \\
\hline
\end{tabular}

\section{SCENARIOS}

The simulation setup was applied to three scenarios. These scenarios are designed to evaluate the network performance in different situations. The first scenario is to test the three reactive protocols-DSR, AODV, and DYMO-when the number of nodes increases. The second scenario is to evaluate the performance of the network in the presence of obstacles. The idea of the third scenario is to simulate a real network. A group of nodes will be shut down suddenly during communication.

\section{A. First Scenario}

In the first scenario, the network performance was tested by using three reactive protocols, when the number of nodes increased from 25 to 45 . The network was implemented in two cases - the first case with 25 nodes and the second case with 45 nodes. Fig. 9 shows the diagram of the network with 25 nodes, which is configured with the specifications listed in Table IV. The source started to send the RREQ to the nodes in its transmission range, where only one node received the RREQ. Fig. 10 shows the network with 45 nodes. The performance of the protocols was evaluated and examined when the number of nodes increased. The idea behind this scenario is to determine which protocol performs well when the number of nodes increases with fast mobility. The network was implemented with a fixed transmitter (sender1) and receiver (reciver1). The rest of the nodes move with a velocity varying from 0.01 to 15 meters per second. Each node has a transmission range coupled with its transmission power and receiving sensitivity.

\section{B. Second Scenario}

MANET is a network that is designed to operate in critical situations such as battlefield, emergency, and rescue missions. Thus, it is important to test the network in difficult situations. This scenario implemented three obstacles that were used to block the signal. The obstacles took the shape of black rocks, as shown in Fig.11. The network has 25 nodes with fast mobility varying from 0.01 to 15 meters per second. Each node has a transmission range coupled with its transmission power and receiving sensitivity. The transmitter and receiver network are fixed nodes in the network. The simulation setup in Table IV was used in this scenario to compare the results 
with those of the first scenario in the case of 25 nodes. This will indicate how obstacles affect network performance.

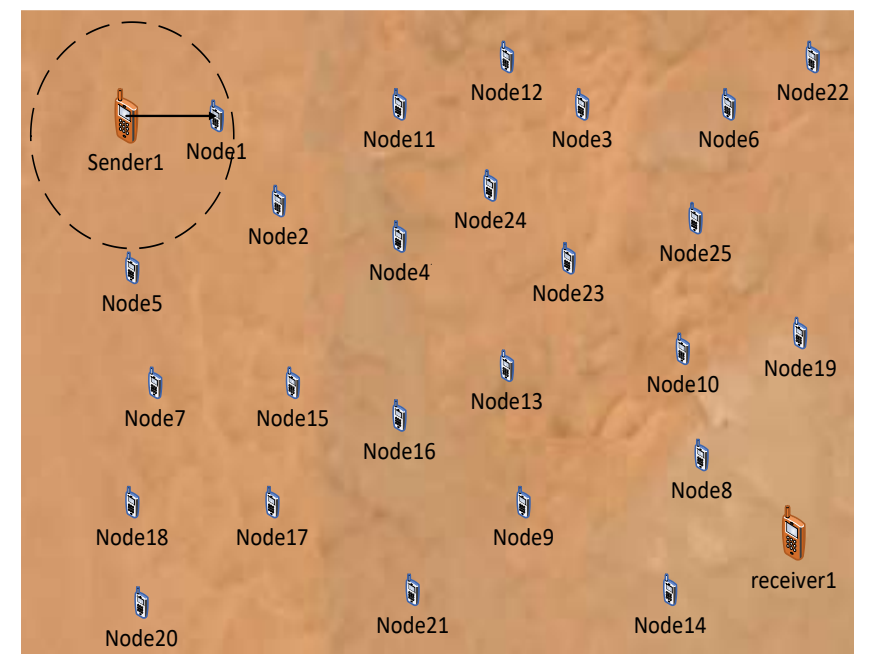

Fig. 9. Network during the Simulation with 25 Nodes.

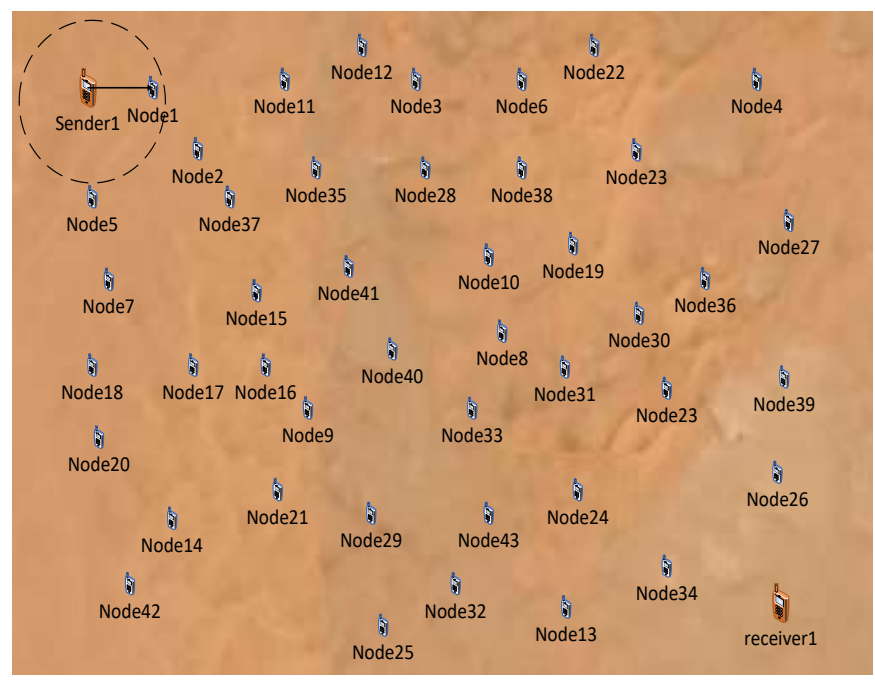

Fig. 10. The Network during Simulation with 45 Nodes.

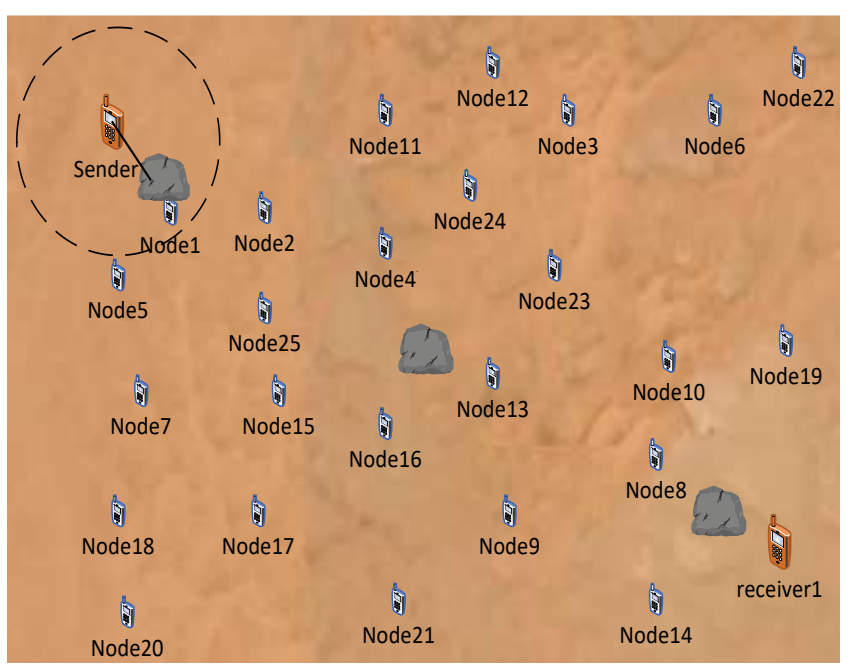

Fig. 11. The Network during Simulation with 3 Obstacles.

\section{Third Scenario}

In this scenario, the network was tested when a group of nodes is shut down suddenly. In the real network, the nodes have a limited battery, which will shut down the node during the communication. The node will shut down due to the battery or may experience any malfunction. In this scenario, the network has 45 nodes. When the simulation time reaches 250s, the group of nodes (Node [33] to Node [44]) will shut down. Therefore, the network must respond quickly and recover the path between the source and the destination. The transmitter and receiver were fixed in the network. The rest of the nodes move with a velocity varying from 0.01 to 15 meters per second. The simulation setup in Table IV was used in this scenario, as well as the first and second scenarios. Fig. 12 shows the nodes that are shut down at 250s.

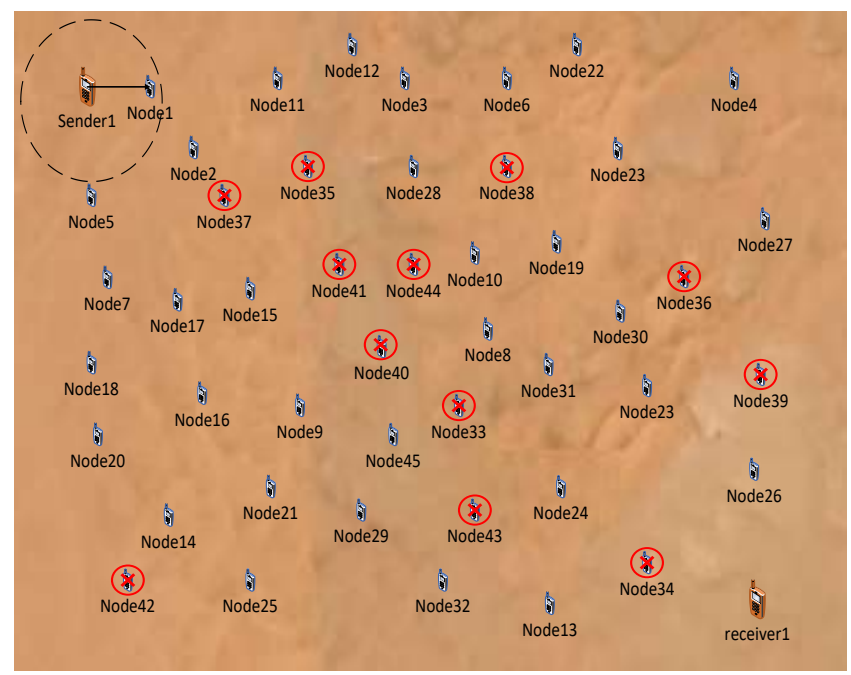

Fig. 12. The Network during Simulation with Shutdown 12 Nodes.

\section{RESULTS}

The network evaluation is carried out in terms of packets received, end-to-end delay, transmission count or routing overhead, throughput, and packet ratio.

\section{A. Packet Received}

Packets received is the number of packets that the destination successfully received. The higher the number of packets received, indicates the effectiveness of the protocol used. Fig. 13 shows the comparison between the three protocols in the first scenario for two cases when the number of nodes increases from 25 to 45 . DYMO is proven to be an efficient protocol when the number of nodes is high, with fast mobility. When the number of nodes is 25, DYMO works better than DSR and AODV. Moreover, when the number of nodes increases to 45 , the number of received packets increases in DYMO. The performance of AODV improves when the number of nodes increases as well. DSR performance was degraded when the number of nodes increases, which means DSR did not work well when the network has high-speed mobility nodes resulting in a high packet drop. Fig. 14 shows the results of the second scenario for the three protocols. The network in the second scenario suffered from packet loss for all three protocols; the loss in packets was due to the obstacles. Thus, the three protocols did 
not perform efficiently in the presence of obstacles. Compared to the first scenario with 25 nodes, DYMO lost 540 packets more, AODV lost 35 more packets, and DSR lost 138 more packets, and all of this was due to the obstacles. Although DYMO saw the biggest decrease in received packets (compared to the first scenario in case of 25 nodes), it still outperformed its peers in the second scenario.

In the third scenario, as shown in Fig. 15, the network reopened quickly and recovered the path between the source and destination by using DYMO. AODV had a moderate performance. DSR had the worst performance as compared to the other protocols. However, all three protocols experience a performance degradation if one compares their performance to the first scenario with 45 nodes. Since the third scenario was fixed with 45 nodes, comparing the third scenario to the second part of the first scenario shows the effect of dropped nodes on received packets. If the protocol is robust, it should be able to quickly recover the connection, which directly results in more packets successfully received.

\section{B. End-to-End Delay}

End-to-end delay is the time interval that it takes to send a packet between the source and destination, including processing and queuing time. A smaller end-to-end delay indicates a fast, high-quality network.

\section{Received Packets}

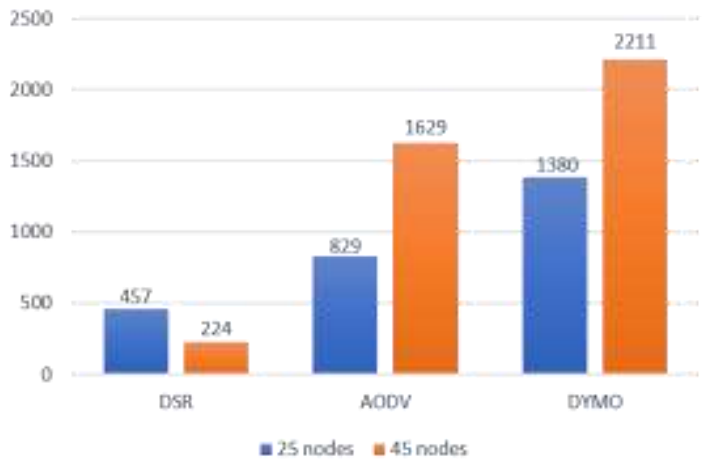

Fig. 13. Received Packets of First Scenario.

\section{Received Packets}

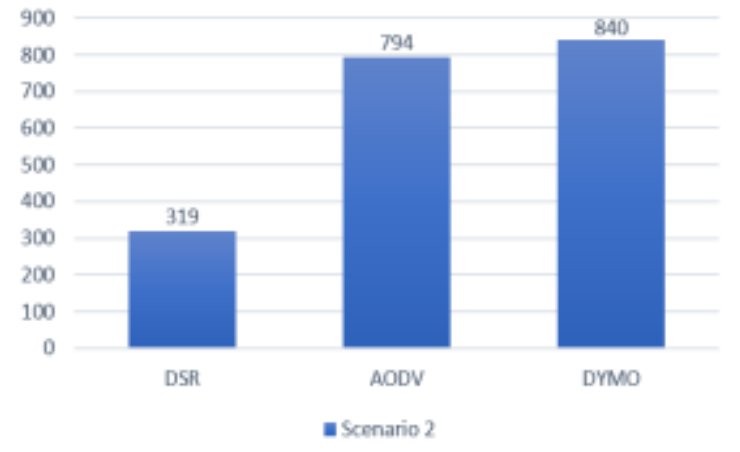

Fig. 14. Received Packets of Second Scenario.

\section{Received Packets}

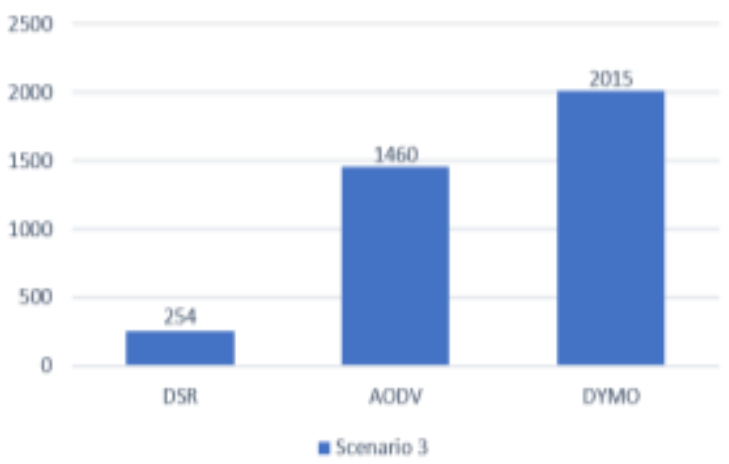

Fig. 15. Received Packets of Third Scenario.

In the first scenario, the three protocols were compared for a varying number of nodes, and the results appear in Fig. 16. In first case, AODV has the smallest end-to-end delay as compared to DYMO and DSR. However, when the number of nodes increases, the end-to-end delay increases by using AODV. DYMO and DSR perform better, in terms of end-toend delay, when the number of nodes increases.

The second and third scenarios are shown in Fig. 17 and Fig. 18. In the second scenario, AODV has the largest end-toend delay, while DSR has the smallest end-to-end delay. DYMO maintains the same performance as compared to the first scenario with 25 nodes, which shows that obstacles did not increase the end-to-end delay with DYMO.

In the third scenario, all three protocols experience an increase in the delay as compared to the first scenario with 45 nodes. The number of nodes decreases suddenly, affecting all three protocols performance. However, AODV has the largest end-to-end delay.

\section{Transmission Count or Routing Overhead}

Transmission count is one of the routing metrics designed for MANETs. It represents the number of transmissions required to send a packet over a link, including the retransmission. A smaller transmission count means less network overhead, which leads to less bandwidth consumption. The results of routing overhead or transmission count are shown in Fig. 19, Fig. 20, and Fig. 21.

\section{End to End Delay}

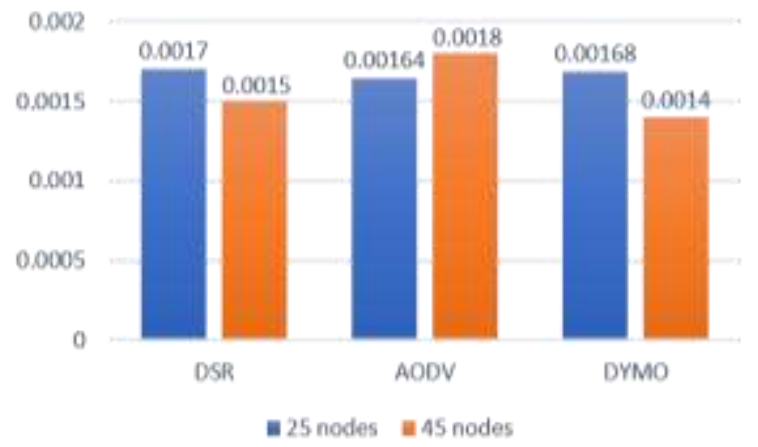

Fig. 16. End to End Delay of First Scenario. 


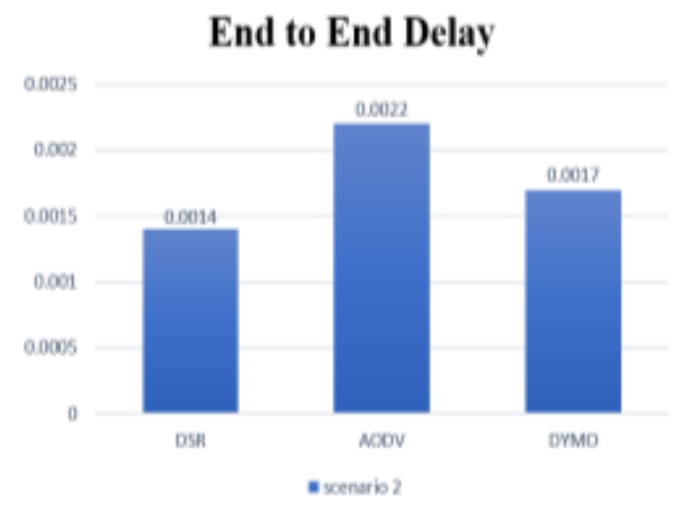

Fig. 17. End to End Delay of Second Scenario.

\section{End to End Delay}

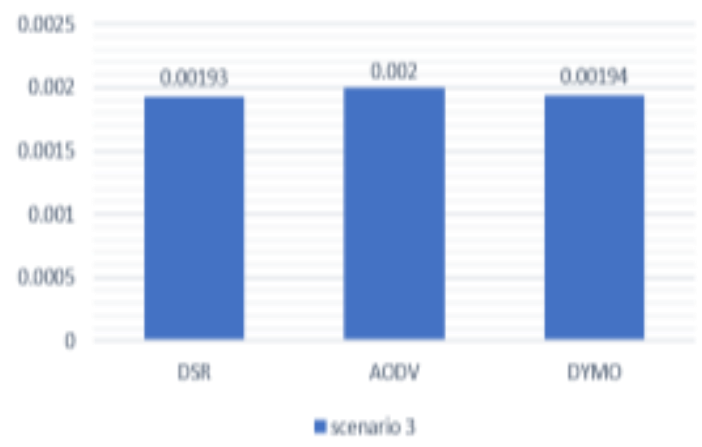

Fig. 18. End to End Delay of Third Scenario.

\section{Transmission count}

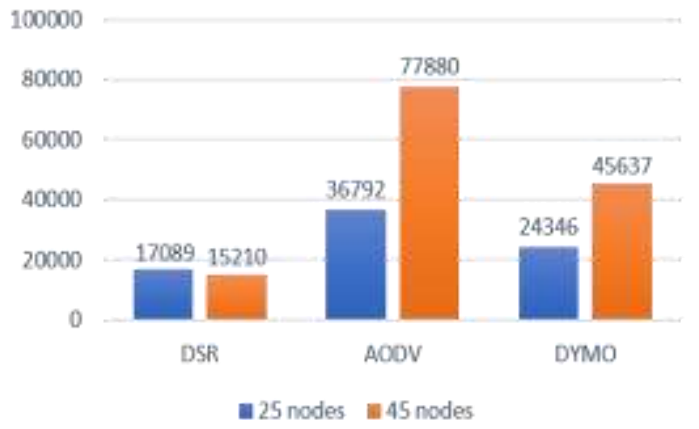

Fig. 19. Transmission Count of First Scenario.

\section{Transmission count}

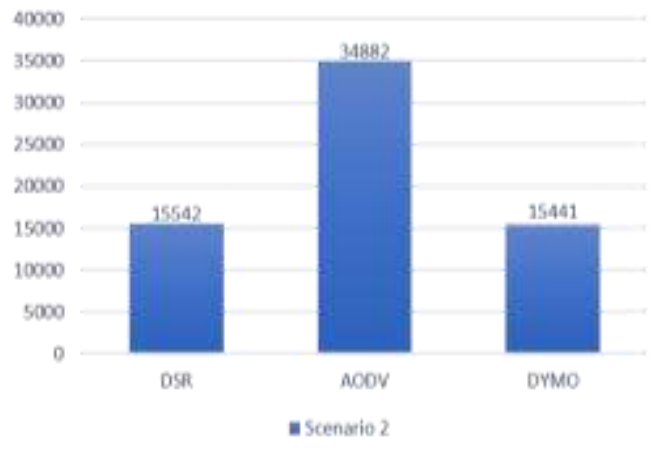

Fig. 20. Transmission Count of Second Scenario.

\section{Transmission count}

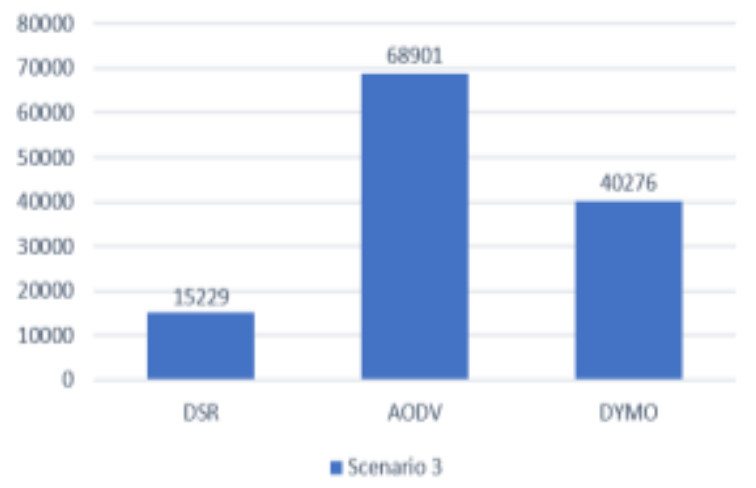

Fig. 21. Transmission Count of Third Scenario.

AODV has the largest transmission count among the three protocols in all three scenarios. AODV uses periodic messages to maintain the routes between the nodes, which increases the routing overhead. DYMO has less routing overhead than AODV, as DYMO uses an accumulation path function. Therefore, DYMO allows the nodes in an active route to use the information of intermediate nodes to the destination to update their routing tables, which helps to reduce the retransmission packets and the RREQ numbers when communication is needed in the future. DSR has the lowest routing overhead except for the second scenario, due to the lack of a periodic message used to maintain the route process. DSR uses an acknowledgment that confirms the link capability to carry the data, which already exists in MAC protocols. This acknowledgement gives DSR an advantage over the other protocols in terms of routing overhead.

\section{Throughput}

Throughput is the number of packets successfully received at the destination per unit of time and is measured in bits per second.

Throughput $=\frac{\sum \text { Number of bit received }}{\text { Simulation time }}$

Throughput is considered the most important metric identifying the quality of a network. Fig. 22 shows that DYMO is an efficient and reliable protocol when the number of nodes increases and have fast mobility. DYMO has the largest throughput in both cases of the first scenario. AODV has an average performance, but the performance increases when the number of nodes increases. DSR has the worst performance; when the number of nodes increases, the throughput decreases.

The second scenario results are shown in Fig. 23. The network suffered from high packet loss in the presence of obstacles. However, DYMO still has the highest performance, while DSR has the worst performance. In the third scenario, as shown in Fig. 24, the use of DYMO will help the network respond and recover the connection between the nodes faster as compared to DSR and AODV. AODV has average performance, while DSR has the worst performance. This leads to the conclusion that DSR is not suitable for difficult situations. 


\section{Throughput}

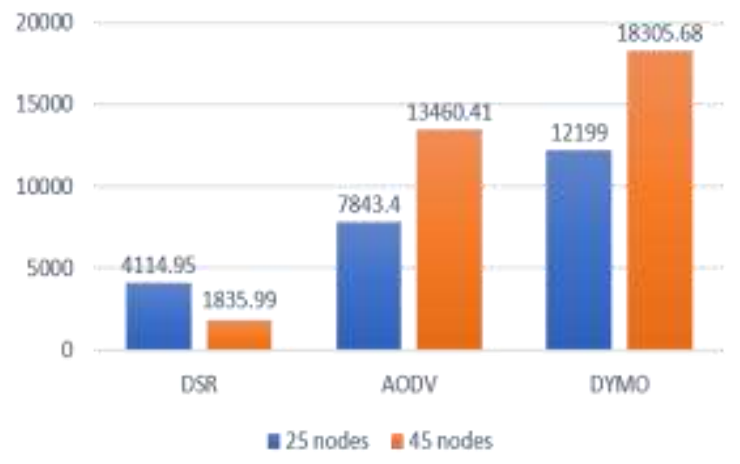

Fig. 22. Throughput of First Scenario.

\section{Throughput}

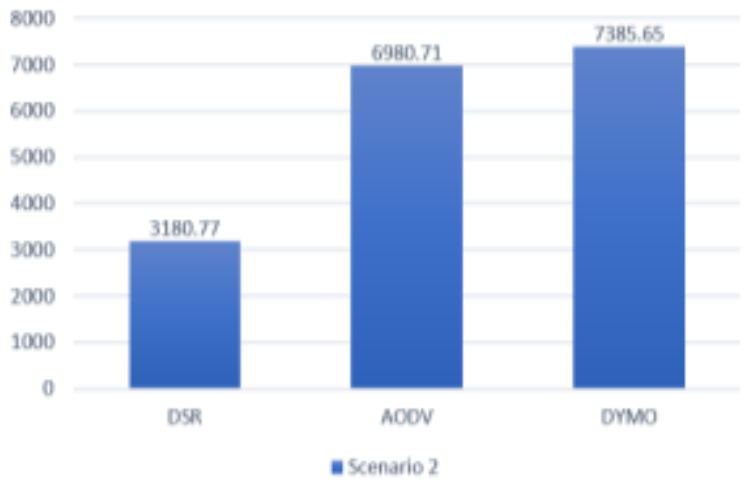

Fig. 23. Throughput of Second Scenario.

\section{Throughput}

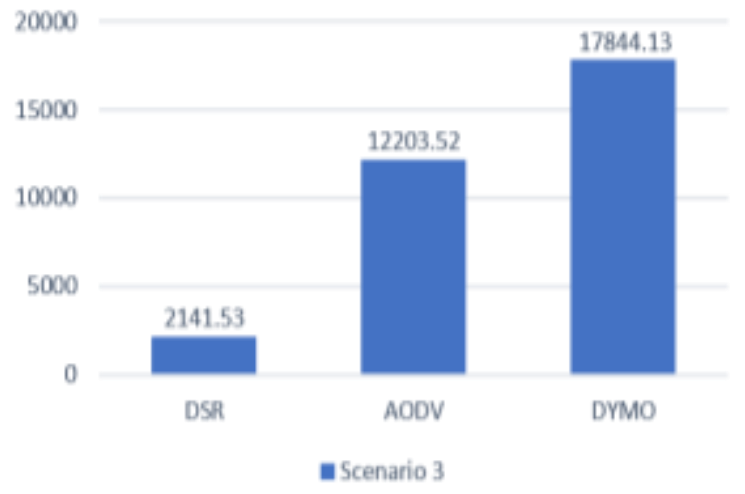

Fig. 24. Throughput of Third Scenario.

\section{E. Packet Ratio}

This is the ratio of the number of data packets delivered to the destination and the total number of data packets sent by the source. DYMO has the largest number of delivered packets, as discussed before in Fig. 13, Fig. 14, and Fig. 15. Therefore, DYMO has the highest packet ratio, making it a more reliable protocol among the three in the case of increasing the number of nodes. Fig. 25, shows the comparison when the number of nodes increases from 25 to 45 .

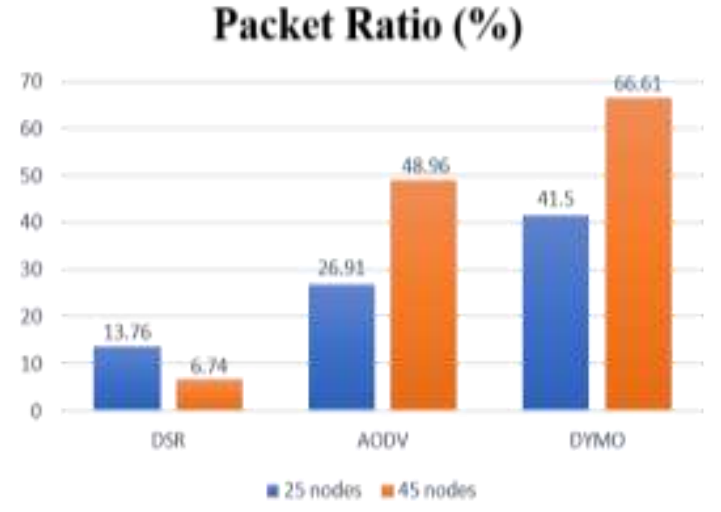

Fig. 25. Packet Ratio of First Scenario.

In the second scenario, as shown in Fig. 26, DYMO has the highest packet ratio of $25.3 \%$, which means $74.7 \%$ of the transmitting packets were lost. AODV received 23.95\% packets from the total number of packets sent by the transmitter node means $76.05 \%$ of transmitting packets were lost, and DSR received only $9.61 \%$ which means $90.39 \%$ of the total packets were lost. In summary, all three protocols did not perform well in the second scenario.

In the third scenario, as shown in Fig. 27, DYMO achieved a packet ratio of $60.7 \%$, which is the largest packet ratio as compared to the other protocols. AODV and DSR achieved packet ratios of $43.88 \%$ and $7.65 \%$, respectively.

\section{Packet Ratio (\%)}

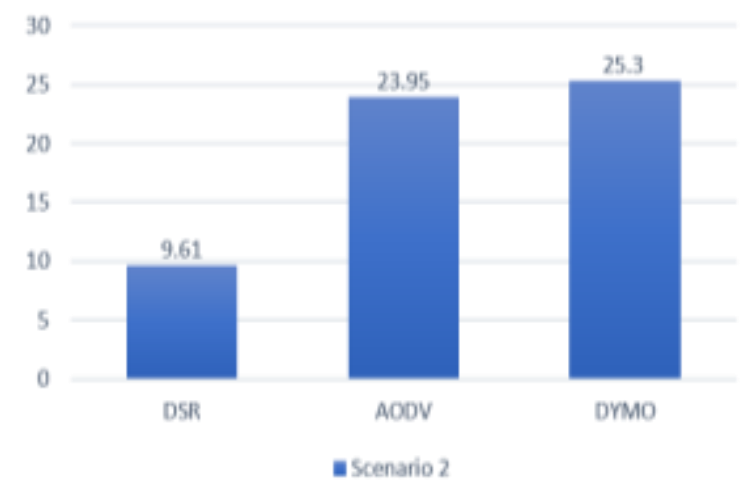

Fig. 26. Packet Ratio of Second Scenario.

\section{Packet Ratio (\%)}

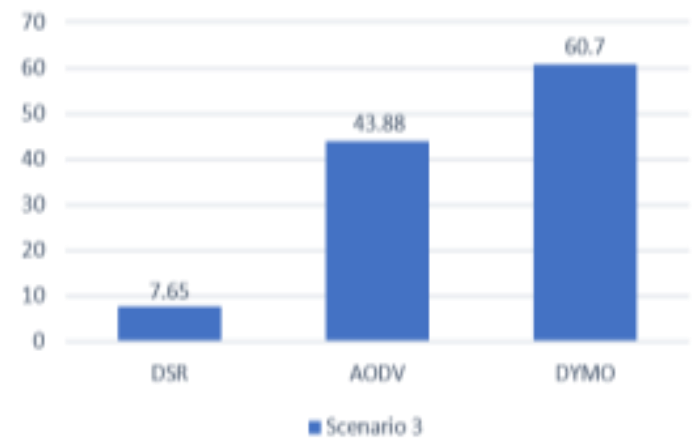

Fig. 27. Packet Ratio of Third Scenario. 


\section{VII.CONCLUSION}

MANETs are used in different applications, especially in critical applications, due to their features and characteristics that make the networks very flexible. In this paper, DYMO proved to be an efficient and reliable protocol compared to DSR and AODV when the network is large and has fast mobility. DSR performs worst when the number of nodes increases. AODV has average performance, but when the number of nodes increases, the performance increases. Also, AODV has the largest routing overhead due to the periodic messages that are used to maintain the link between the nodes in the active route. Overall, DYMO has the highest performance in the three scenarios and works better under challenging situations than DSR and AODV.

\section{REFERENCES}

[1] H. Valchanov, J. Edikyan, and V. Aleksieva, "An Empirical Study of Wireless Security in City Environment," in Proceedings of the 9th Balkan Conference on Informatics, 2019, pp. 1-4.

[2] M. A. Jubair et al., "Competitive Analysis of Single and Multi-Path Routing Protocols in Mobile Ad-Hoc Network," Indones. J. Electr. Eng. Comput. Sci., vol. 14, no. 2, 2019.

[3] A. M. Abdullah, E. Ozen, and H. Bayramoglu, "Energy Efficient MANET Routing Protocol Based on Ant Colony Optimization.," Adhoc Sens. Wirel. Networks, vol. 47, 2020.

[4] M. Sharma, M. Singh, K. Walia, and K. Kaur, "Comprehensive Study of Routing Protocols in Adhoc Network: MANET," in 2019 IEEE 10th Annual Information Technology, Electronics and Mobile Communication Conference (IEMCON), 2019, pp. 792-798.

[5] T.-A. N. Abdali, R. Hassan, R. C. Muniyandi, A. H. Mohd Aman, Q. N. Nguyen, and A. S. Al-Khaleefa, "Optimized Particle Swarm Optimization Algorithm for the Realization of an Enhanced EnergyAware Location-Aided Routing Protocol in MANET," Information, vol. 11, no. 11, p. 529, 2020.

[6] A. M. Shantaf, S. Kurnaz, and A. H. Mohammed, "Performance Evaluation of Three Mobile Ad-hoc Network Routing Protocols in Different Environments," in 2020 International Congress on HumanComputer Interaction, Optimization and Robotic Applications (HORA), 2020, pp. 1-6.
[7] V. Kumar, "Improving quality of service in mobile ad-hoc networks (MANETs) using adaptive broadcast scheduling algorithm with dynamic source routing protocol," J. Comput. Theor. Nanosci., vol. 14, no. 9, pp. 4370-4376, 2017.

[8] P. Nayak and B. Vathasavai, "Impact of random mobility models for reactive routing protocols over MANET," Int. J. Simulation--Systems, Sci. Technol., vol. 17, no. 34, pp. 112-115, 2016.

[9] I. Nurcahyani and F. F. Laksono, "Performance Analysis of Ad-Hoc OnDemand Distance Vector (AODV) and Dynamic Source Routing (DSR) Routing Protocols During Data Broadcast Storm Problem in Wireless Ad Hoc Network," in 2019 International Seminar on Intelligent Technology and Its Applications (ISITIA), 2019, pp. 29-34.

[10] R. Rana and R. Kumar, "Performance Analysis Of Aodv In Presence Of Malicious Node," Acta Electron. Malaysia, vol. 3, no. 1, pp. 1-5, 2019.

[11] Bisoyi, S.K.; Sahu, S. Performance analysis of Dynamic MANET Ondemand (DYMO) Routing protocol. Spec. Issue IJCCT 2010, 1, 3.

[12] U. Yavuz, F. A. Dael, and W. A. J. S. A. Jabbar, "Performance Evaluation of DYMO and OLSRv2 Routing Protocols in VANET," Int. J. Integr. Eng., vol. 12, no. 1, pp. 50-58, 2020.

[13] Jamilah Alamri, Amnah S. Al-Johani, K. Ibrahim. Ata, "Performance Evaluation of Two Mobile Ad-hoc Network Routing Protocols: Ad- hoc On-Demand Distance Vector, Dynamic Source Routing”, IJAST, vol. 29, no. 05, pp. 9915 - 9920, May 2020.

[14] R. Skaggs-Schellenberg, N. Wang and D. Wright, "Performance Evaluation and Analysis of Proactive and Reactive MANET Protocols at Varied Speeds," 2020 10th Annual Computing and Communication Workshop and Conference (CCWC), Las Vegas, NV, USA, 2020, pp. 0981-0985.

[15] M. H. Hassan et al., "Mobile ad-hoc network routing protocols of timecritical events for search and rescue missions," Bull. Electr. Eng. Informatics, vol. 10, no. 1, pp. 192-199, 2020.

[16] A. S. Mustafa, M. M. Al-Heeti, M. M. Hamdi, and A. M. Shantaf, "Performance analyzing the effect of network size on routing protocols in manets," in 2020 International Congress on Human-Computer Interaction, Optimization and Robotic Applications (HORA), 2020, pp. $1-5$.

[17] D. S. Som and D. Singh, "Performance analysis and simulation of AODV, DSR and TORA routing protocols in MANETs," Int. J. Recent Technol. Eng., vol. 1, no. 3, pp. 2277-3878, 2012. 